\title{
Influence of Domain-General Abilities and Prior Division Competence on Fifth-Graders' Fraction Understanding
}

\author{
Florencia Stelzer ${ }^{1 *}$, María Laura Andrés ${ }^{1}$, Lorena Canet-Juric ${ }^{1}$, Sebastián Urquijo 1 , \\ María Marta Richards ${ }^{1}$ \\ ${ }^{1}$ Institute of Basic, Applied Psychology and Technology (IPSIBAT), National University of Mar del Plata, National Scientific and \\ Technical Research Council (CONICET), Mar del Plata, ARGENTINA \\ *CORRESPONDENCE: $\bigotimes$ florenciastelzer@gmail.com
}

\begin{abstract}
The present study analyzed the relationship between domain-general abilities and fraction knowledge in fifth grade, and investigated the mediating role of division competence in that relationship. Children $(n=175)$ were assessed in fourth grade on domain-general abilities (selective attention, working memory, fluid intelligence) and on division competence; and in fifth grade on fraction conceptual knowledge. Mediation analyses revealed that domain general abilities were direct predictors of fraction concepts, and division competence mediates the $32 \%$ of the effect of working memory and the $17 \%$ of the effect of intelligence on fraction knowledge. These findings support the assumptions of those theoretical models of numerical cognition that proposed a central role of general cognitive abilities for mathematics learning and indicate that there are distinct pathways from general cognitive abilities to fraction conceptual knowledge.
\end{abstract}

Keywords: fraction learning, cognitive abilities, math cognition, rational numbers, math learning

\section{INTRODUCTION}

Fraction knowledge is of particular importance in educational mathematics (DeWolf, Bassok, \& Holyoak, 2015; 2016; Torbeyns, Schneider, Xin, \& Siegler, 2014). The integrated theory of numerical development suggests that its learning improves the students' comprehension on numeral systems and operations, contributing to the process of learning more complex mathematical concepts (Siegler \& Lortie-Forgues, 2017; Siegler, Thompson, \& Schneider, 2011). There is consistent empirical evidence indicating that fraction understanding constitutes one of the most important predictors of learning more advanced mathematical content, such as algebra (Booth \& Newton, 2012; Booth, Newton, \& Twiss-Garrity, 2014; DeWolf et al., 2015; 2016; Siegler et al., 2012).

Fraction learning represents a challenge for students, as they have to include a series of concepts in opposition to their previous knowledge of whole numbers (e.g., fractions allow to represent a magnitude through different expressions, fractions composed with greater number of digits do not always have a higher value, etc.). This leads to a significant number of students experiencing difficulties in their comprehension (Carpenter, Corbitt, Kepner, Lindquist, \& Reys, 1980; Chan, Leu, \& Chen, 2007). In the context of Latin America, for instance, the results of the Third Regional Comparative and Explanatory Study (TERCE in Spanish) (Flotts, Manzi, Jiménez, Abarzúa, Cayuman, \& García, 2016) suggested that only 5.1\% of the representative sample of students of sixth grade was capable of solving complex problems with fractions. Similar problems in fraction learning were also reported in students from Asia, Europe and North America

Article History: Received 25 January $2019 \bullet$ Revised 19 February $2019 \bullet$ Accepted 21 February 2019

(C) 2019 by the authors; licensee Modestum Ltd., UK. Open Access terms of the Creative Commons Attribution 4.0 International License (http://creativecommons.org/licenses/by/4.0/) apply. The license permits unrestricted use, distribution, and reproduction in any medium, on the condition that users give exact credit to the original author(s) and the source, provide a link to the Creative Commons license, and indicate if they made any changes. 
(e.g., Carpenter et al., 1980; Chan et al., 2007; Gabriel, Coché, Szucs, Carette, Rey, \& Content, 2013), denoting that difficulties in fraction understanding occur in many educative systems.

Several theoretical models and empirical studies have indicated that general cognitive abilities (e.g., attention, intelligence) constitute central variables during mathematics learning (Geary 2004; Geary et al., 2008; Lefevre et al., 2010). As regards fractions, the contribution of selective attention, working memory and fluid intelligence to explain fractions has been mainly considered in the literature (Hansen et al., 2015; Jordan et al., 2013). Selective attention -also known as access or perceptual inhibition- is the capacity to focus attention on relevant information while suppressing the interference from the irrelevant one (Diamond, 2013; Hasher, Lustig, \& Zacks, 2007). This capacity prevents irrelevant information from gaining access to working memory, allowing the controlled information processing to achieve goals (Hasher et al., 2007).

Working memory constitutes a limited capacity system involved in the active storage and processing of information during short temporary periods of time (Miyake \& Shah, 1999). The working memory model of Baddeley and Hitch (1974) constitutes one of the most accepted models in the literature. Its first version distinguishes three components: two slave systems -involved in the active storage of information- and a central executive, responsible for manipulating, updating and monitoring interferences during information storage (Baddeley \& Hitch, 1974; Baddeley, 2012). This last component is considered the nucleus of working memory (Engle, 2002; Engle \& Kane, 2004, in Peng, Barnes, Namkung \& Sum, 2016), as it controls and coordinates the active storage and processing of information in the other components of this system.

Finally, fluid intelligence is the capacity to deliberately use mental operations with the purpose of solving new problems (those in which overlearned abilities and prior knowledge are not appropriate) (Cattell, 1971). Those mental operations include producing inferences, developing concepts, classifying, generating, and contrasting hypothesis, identifying relationships and transforming information (McGrew, 2009; McGrew \& Evans, 2004; Kane \& Gray, 2005).

Fraction learning is mainly developed by solving problems that require identifying the relevant information and suppressing the irrelevant one, representing, manipulating, and updating number and/or spatial relationships among variables, identifying and abstracting number relationships among factors, making inferences, and contrasting hypothesis. Therefore, it is expected that selective attention, working memory, and fluid intelligence contribute to this learning. Nonetheless, the empirical evidence shows different results regarding the contribution of these cognitive abilities to fraction knowledge and there are some controversies regarding the direct or mediated nature of their relation.

Concerning the direct effects of cognitive abilities on fraction knowledge, Jordan et al. (2013) reported that student's attentional capacity in third grade predicted fraction conceptual knowledge in fourth grade; while Namkung and Fuchs (2015) did not find a significant contribution of attention capacity at the beginning of fourth grade to fraction conceptual knowledge at the end of fourth grade. Regarding working memory, some authors indicated that this process in first (Bailey, Siegler \& Geary, 2014) and fifth (Hansen et al., 2015) grade predicted fraction conceptual knowledge in sixth (Hansen et al., 2015) and eighth grade (Bailey et al., 2014). Nevertheless, other authors have not observed that working memory explained fraction knowledge in fourth grade (Jordan et al., 2013; Namkung \& Fuchs, 2015). Finally, in relation to fluid intelligence, Vukovic et al. (2014) suggested that this ability in first grade did not explain fraction conceptual knowledge in fourth grade; nonetheless, other researchers found that this capacity in third and fourth grade predicted fraction conceptual knowledge in fourth grade (Jordan et al., 2013; Namkung \& Fuchs, 2015). The complexity of fraction knowledge required in different school grades varies, so it is possible that the differing results among studies are due to their differences in the school grade analyzed.

On the other hand, learning mathematics shows a hierarchical structure, where the consolidation of the simplest knowledge supports the development of more complex concepts and abilities (Geary et al., 2008; Locuniak \& Jordan, 2008). There is empirical evidence indicating that attention, working memory and fluid intelligence are related to prior math knowledge that is necessary to understand fractions (e.g., whole number calculation ability) (Siegler \& Pyke, 2013; Hansen et al., 2015; van der Ven, van der Maas, Straatemeier, \& Jansen, 2013). Therefore it could be possible that attention, working memory and fluid intelligence affect fraction knowledge indirectly, through their effect on the prior math knowledge that is required to learn fractions (mediated relation). In such case, prior math knowledge serves as an intermediary (mediator) in the relation between those cognitive abilities and fraction knowledge. Mediators are those intermediate variables that represent the generative mechanisms through which the focal independent variable is able to influence the dependent variable of interest (Baron \& Kenny, 1986). 
Empirical studies about indirect relation between cognitive abilities and fraction knowledge are scarce. Vukovic et al. (2014) showed that attentional capacity in first grade was only indirectly related to fraction knowledge in fourth grade, through the effect produced by attention on whole number calculation ability in second grade. Additionally, Ye, Resnick, Hansen, Rodrigues, Rinne, and Jordan (2016) reported that attention, working memory and fluid intelligence in third grade indirectly affect fraction conceptual knowledge in sixth grade, being the relationship fully mediated by magnitude reasoning ability in fifth grade. Nonetheless, contrary to Vukovic et al. (2014), Ye et al. (2016) did not observe any mediating effect of general whole number calculation ability in fifth grade in the relation between general cognitive processes (attention, working memory and fluid intelligence) and fraction conceptual knowledge in sixth grade. On balance, the results of Vukovic et al. (2014) and Ye et al. (2016) suggest that the effect of general cognitive abilities on fraction knowledge could be mediated by prior math knowledge, and mediators varies according the school grade analyzed.

Division ability can be considered as a possible mediator of the effect of cognitive abilities on fraction knowledge. There is empirical evidence indicating that attention, working memory and fluid intelligence are related to division ability (Siegler \& Pyke, 2013; Hansen et al., 2015; van der Ven et al., 2013), and division ability is theoretically and empirically related to fraction knowledge (Hansen et al., 2015; Sielger \& Pyke; 2013). Fractions a/b expression basically shows a division and it is used to express the quotient of a splitting operation (e.g., "If a piece of chocolate is distributed in equal parts among four children, each child will receive $1 / 4$ "). Based on the foregoing, initial fraction teaching is usually scaffold on division knowledge (General Management of Culture and Education, 2018). Due to the above, it is possible that the relation of selective attention, working memory and fluid intelligence to fraction knowledge occurs both directly and via their effect on division ability (indirect relation).

Studying the direct and indirect effects of cognitive abilities on fraction learning could provide a more comprehensive model of fraction understanding. Therefore, the objective of this study is to analyze the relationship of selective attention, working memory, and fluid intelligence on fraction knowledge in fifth grade of Argentinean elementary school, and also to establish if this relation is mediated by division ability. This school grade is of interest because it represents an intermediate point in the process of fraction teaching in the educative system of Argentina (Ministry of Education, 2011). In this educative system, the systematic teaching of fractions is introduced in fourth grade, mainly by working with sharing or partitioning problems linked to division and which solution involves common fraction expressions (e.g., "Four brothers share three pizzas equally, how much of a pizza does each brother get?") (General Management for Culture and Education, 2018). Later in fifth and sixth grade students must mainly work with more complex fraction problems (e.g., number line estimation problems and equivalence recognition problems with uncommon fractions expressions and decimal numbers) (General Management for Culture and Education, 2018). This means that fifth grade represents a leap in the complexity of fraction knowledge required, and this leap might be supported by division knowledge in fourth grade. On the other hand, fifth grade fraction learning is a highly cognitive demanding process, so we expect that the three cognitive processes considered in this study to be directly related to fraction knowledge. In summary, we assume that selective attention, working memory and fluid intelligence will be related to fractions knowledge both directly and indirectly, via division ability.

\section{METHOD}

\section{Participants}

A correlational non-experimental design with a mediation hypothesis was conducted. Participants has been part of a prior study that analyzed the cognitive predictors of division knowledge in fourth grade (Stelzer, Introzzi, Andres, Richard's, \& Urquijo, 2018). The sample of that study was 175 fourth graders. They came from three public and a private school located in urban areas in the city of Mar del Plata, Argentina and they were chosen on a non-probabilistic basis, according to the availability. The present study differ from our prior one (Stelzer et al., 2018) in the objectives and the measures. The objective of the prior study was to analyze the cognitive predictors of division knowledge in fourth grade, fraction knowledge was not assessed in that study because it was not a variable of interest. The present study goal is to analyze the relationship of selective attention, working memory, and fluid intelligence on fraction knowledge in fifth grade and also to establish if this relation is mediated by division ability. To archive this goal we followed the participants of Stelzer et al. (2018) and we assessed their fraction knowledge in fifth grade. 
Only 152 students of Stelzer et al. (2018) participated in fifth grade. The reason for the discontinuity of the 23 other participants was change of school $(n=11)$, or else unknown $(n=12)$. Out of the 152 students that participated in fifth grade, those cases that presented one of the following conditions were excluded: (a) previous diagnosis of neurological or mental disorders, (b) birth weight under 2.5 kilograms or born prior to the 37th week of gestation, (c) score corresponding to the deficient category in the Raven's Colored Progressive Matrices Test (1989) (Cayssials, 1993), (d) being under a diagnosis stage, or psychological, psychiatric, or psycho-pedagogical treatment, and (e) absence of school education on fractions during fifth grade. The final sample, whose results are the ones reported in this study, included 97 participants (44 boys and 53 girls) of different social status (SS) (15.6\% low; $21.9 \%$ medium-low; $17.2 \%$ medium; $21.9 \%$ medium-high; $23.4 \%$ high).

\section{Measures}

\section{Health record}

It is a questionnaire based on a parental report exploring the variables (a) weeks of pregnancy at birth, (b) birth weight, (c) neurological or mental disorder diagnosis before the study development, and (d) diagnostic evaluation, or ongoing psychological, psychiatric, or psycho-pedagogic treatment.

\section{Social status questionnaire}

It consists in a semi-structured questionnaire that assess educational level and type of occupation of the main child breadwinner. Social status was computed using the Hollingshead Index (2011). For its estimation, the educative level of both parents was defined according to the scale proposed for Argentina by Pascual, Galperín and Bornstein (1993), and the occupation level was defined according to the Sautús EGO 70 social prestige scale (1989).

\section{Selective attention}

We used the Conjunctive Visual Search Task (CVST) from the Cognitive Self-Regulation Test Battery (TAC in Spanish) (Introzzi \& Canet Juric, 2013) and the Perception of Differences Test (FACES-R) (Thurstone \& Yela, 2012). The CVST is a computerized test in which the participant must identify the presence or absence of a target stimulus (blue square) among a variable set of stimuli that act as distracters (4, 8, 16 and 32 stimuli). The distracters share one of the target's two perceptual traits: color or form. This fosters visual similarity among stimuli and generates the interference effect required in order to activate selective attention. The task is composed by 10 practice trials, followed by 120 experimental trials. The experimental trials are equally distributed in four conditions defined by the number of distractors $(4,8,16$, and 32). The conditions are randomly distributed along the experimental trials. In each trial the participant must press two different keys $(Z$ and $M)$ to indicate whether the target stimulus is present $(Z)$ or absent $(M)$. In every trial half of the distractors are blue circles and the other half are red squares. The percentage of correct responses in the condition with 32 distractors (condition of higher interference and greater attentional demand) was used as indicator of selective attention ability. Previous studies indicated that this task presents appropriate indexes of reliability and validity in children (Richards, Introzzi, Zamora, \& Vernucci, 2017; Richards, Vernucci, Zamora, Canet Juric, Introzzi, \& Guardia, 2017).

The Perception of Differences Test (FACES-R) (Thurstone \& Yela, 2012) constitutes a paper-and-pencil test that shows 180 graphical elements consisting in schematic drawings of faces with mouth, eyes, eyebrows and hair. These drawings are grouped within rectangles containing three elements (a total of 60 rectangles). Two of the faces within each rectangle are equal, and the task of the participant is to identify and cross out the different face within 3 minutes. The test was administered in groups according to the protocol suggested by Thurstone and Yela (2012). The number of correct responses in 3 minutes was considered as indicator of selective attention capacity. This test shows adequate levels of reliability and validity for its application to school children (Thurstone \& Yela, 2012).

\section{Working memory}

Two dual working memory computerized tasks were administered, one verbal task and one visuospatial task. Dual tasks show the central executive capacity to control the interferences and to coordinate the active storage and processing of information in the other components of working memory. Both dual tasks belong to the Cognitive Self-Regulation Tasks Battery (TAC) (Introzzi \& Canet Juric, 2013) and require that the participant simultaneously execute two activities: a primary and a secondary one. In the case of the verbal dual task, the primary activity requires to remember a series of numbers that appear on the screen one at a 
time, written in different colors. The secondary activity demand that the participants say aloud the color in which each number was presented immediately after the number disappears.

On the other hand, the primary activity of the visuospatial dual task is to remember the sequence of appearance of crosses with different colors presented one at a time on a $4 \times 4$ matrix. In the secondary activity the participants must indicate the color of each cross, using the mouse to point it among a range of colors presented on the screen after each cross disappears.

In both dual tasks the number of stimuli to remember in the primary activities increases by one at a time after correctly remembering the stimuli sequence showed. In the case the participant cannot remember the sequence correctly, another trial is presented, but with the same difficulty. Both tasks are interrupted after two subsequent incorrect trials. The maximum number of stimulus recalled in each primary activity was taken as an indicator of WM capacity. Both tasks (verbal and visuo-spatial) show adequate validity and reliability levels for their application in children (Canet Juric, Introzzi, \& Burin, 2015).

\section{Fluid intelligence}

It was evaluated by Raven's Colored Progressive Matrices Test (Raven, 1989), which enables to measure fluid intelligence capacity through shape comparison and analogical reasoning. It is composed by 36 sheets showing graphical matrices with a missing piece. The participants must identify among six options, the one that completes the matrix. The participants receive one point per each correct response. This task shows adequate levels of validity and reliability (Raven, 1989).

\section{Division ability}

It was used and adaptation of the task performed by Hansen et al. (2015) and Siegler and Pyke (2013) in students of fifth, sixth and eighth grade (students between 10 and 14 years old) in the United States. The task was adapted according to the consolidated knowledge of division expected for fourth grade in the Argentine Educative System (Ministry of Education, 2011). It consists in a paper-and-pencil test in which six divisions of growing complexity are presented consecutively and in independent lines $(9 / 3 ; 10 / 4 ; 56 / 8 ; 180 / 60 ; 1200 / 400$; 306/9). Two points are awarded for each correct response, and one point if the response is correct, but there is a mistake detected in the process of calculating it. The reliability was estimated by the Cronbach's alpha coefficient $(\alpha=.82)$.

\section{Conceptual knowledge of fractions}

It consists in a paper-and-pencil test designed according to the activities proposed in textbooks and school documents of math showing the content of the current curricular design in the Argentine Educative System (Ministry of Education, 2011). The task is consistent with the tasks used in previous studies to measure fractions knowledge in students of fourth and fifth grade (9-11 years old) (e.g., Hecht \& Vagi, 2010; Jordan et al., 2013). It includes 36 items evaluating the different dimensions of the concept of fraction, of which 12 items involve the concept of fractions as a part-whole relation (e.g., "Shade $1 / 2$ on the figure of a rectangle fragmented in 10 equal parts"), 10 items measure the concept of the fractions as a ratio (e.g., "Indicate which of the two fractions presents a higher value"), 10 exercises evaluate fractions as a way to measure magnitude (e.g., "Locate 7/3 in a number line from 0 to 3"), 2 exercises evaluate the concept of fractions as an operator function (e.g., "Calculate $3 / 7$ of 42 ") and 2 as a quotient (e.g., "Six friends bought three pizzas and they divided it in equal parts. How much pizza did each of them eat?"). The participants received 1 point per each correct response. The total number of correct responses was considered as an indicator of performance. The reliability of this task resulted high $(\alpha=.80)$

\section{Proceeding and Ethical Considerations}

In fourth grade the parents were requested to authorize their children to participate by signing an informed consent. The children were informed and the ones having their parents' informed consent and expressing their will to participate were evaluated. The children were evaluated during school time, at the educational institutions they attended, in classrooms prepared for this purpose. Throughout the fourth grade the children were administered tasks of selective attention, working memory, fluid intelligence, and division ability. The conjunctive visual search (selective attention) and the dual working memory (verbal and visuo-spatial) tasks were administered in a sole session of individual evaluation, while the FACES Test (selective attention) and the evaluations about fluid intelligence and division ability were administered in groups during independent evaluation sessions, at the ordinary classroom. The questionnaire on social status and the health record were 
Table 1. Descriptive statistics of cognitive and mathematics factors

\begin{tabular}{lcccc}
\hline & Min. & Max & M & SD \\
\hline Fraction knowledge & 0 & 31 & 7.81 & 6.76 \\
\hline Division ability & 0 & 12 & 5.09 & 3.16 \\
\hline Selective attention (conjunction visual search) & 53.33 & 100 & 85.82 & 10.06 \\
\hline Selective attention (CARAS test) & 15 & 50 & 32.97 & 8.46 \\
\hline Working memory (visoespacial dual task) & 2 & 7 & 3.6 & 1.31 \\
\hline Working memory (verbal dual task) & 2 & 5 & 3.31 & .87 \\
\hline Fluid intelligence & 24 & 36 & 30.46 & 3.33 \\
\hline
\end{tabular}

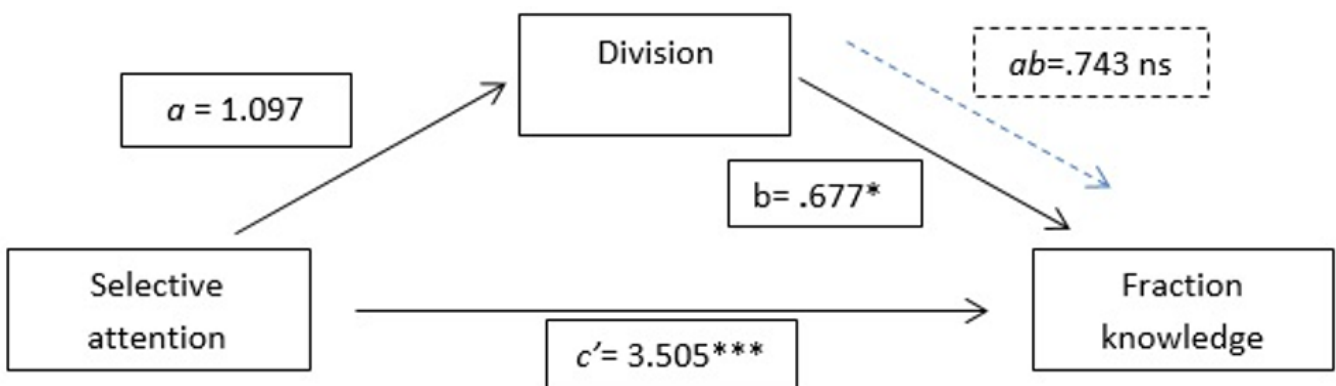

Figure 1. Model testing the mediating effect of division ability on the relationship between selective attention and fraction knowledge

Notes: solid line: direct relation; dashed line: mediated relation

$* * * \mathrm{p}<.001 ; * * \mathrm{p}<.01 ; * \mathrm{p}<.05$

sent to the parents in fourth grade by the school communication notebook. Fraction knowledge was assessed during the second quarter of the fifth grade, in groups, at the ordinary classroom. Before evaluating the students' ability on fractions (fifth grade), we verified, by interviewing the teachers, if the students had received instructions and a school evaluation on the contents involved in this task.

\section{Data Analysis Plan}

Firstly, descriptive analyses on the variables under study were performed (minimal and maximum scores, means and standard deviations) (see Table 1). As two independent indicators were considered for the estimation of both the selective attention capacity and working memory, a common factor was extracted to measure each cognitive ability, and then those factors were used in subsequent analyses.

Secondly, to discard any differences in fraction knowledge according to sex or social status, two parametric tests were performed (t-test and ANOVA for one factor). Several studies have shown that these variables influence mathematics learning (e.g. Brown, 1991).

Subsequently, three mediation models were made. These models included the capacity of division as a mediator of the effect produced by cognitive abilities on fraction knowledge.

\section{Results}

\section{Descriptive analysis}

Table 1 shows the descriptive statistics of the variables under study.

\section{Differences in sex and social status}

No differences were found in fraction knowledge according to sex (t $(91)=-1.38, \mathrm{p}=.17)$ or social status $(\mathrm{F}(5,75)=1.46, \mathrm{p}=.21)$.

\section{Mediation analysis}

Mediation analysis showed a direct effect of selective attention, working memory and fluid intelligence on fraction knowledge. The mediation analysis indicated a significant indirect effect of working memory and fluid intelligence on fraction knowledge, produced via division ability (Figures 1-3). 


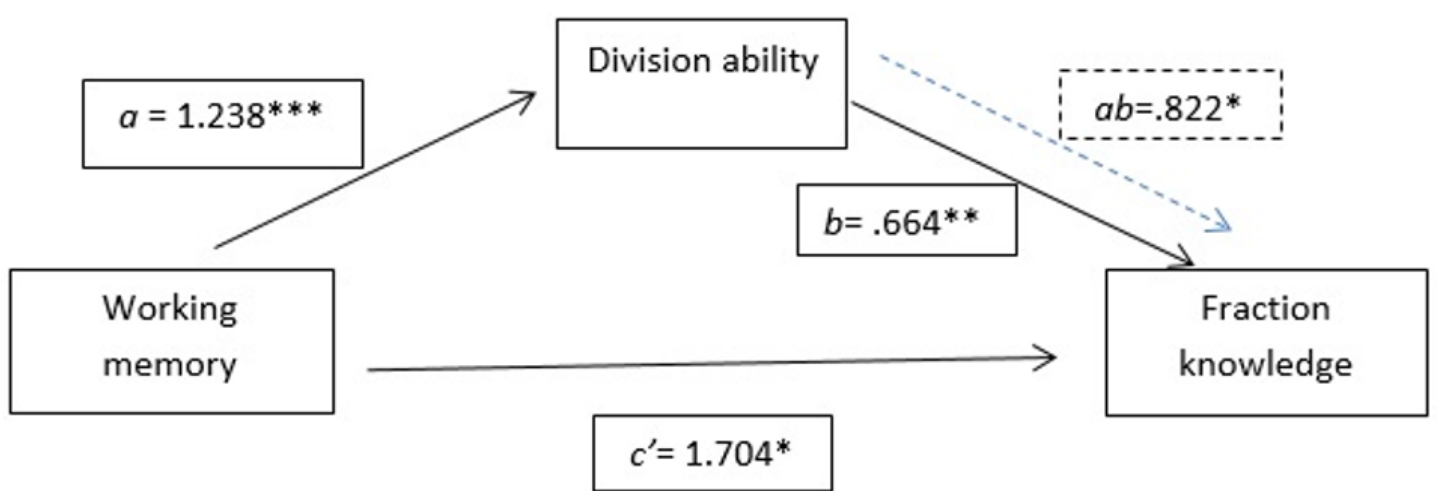

Figure 2. Model testing the mediating effect of division ability on the relationship between working memory and fraction knowledge

Notes: solid line: direct relation; dashed line: mediated relation $* * * \mathrm{p}<.001 ; * * \mathrm{p}<.01 ; * \mathrm{p}<.05$

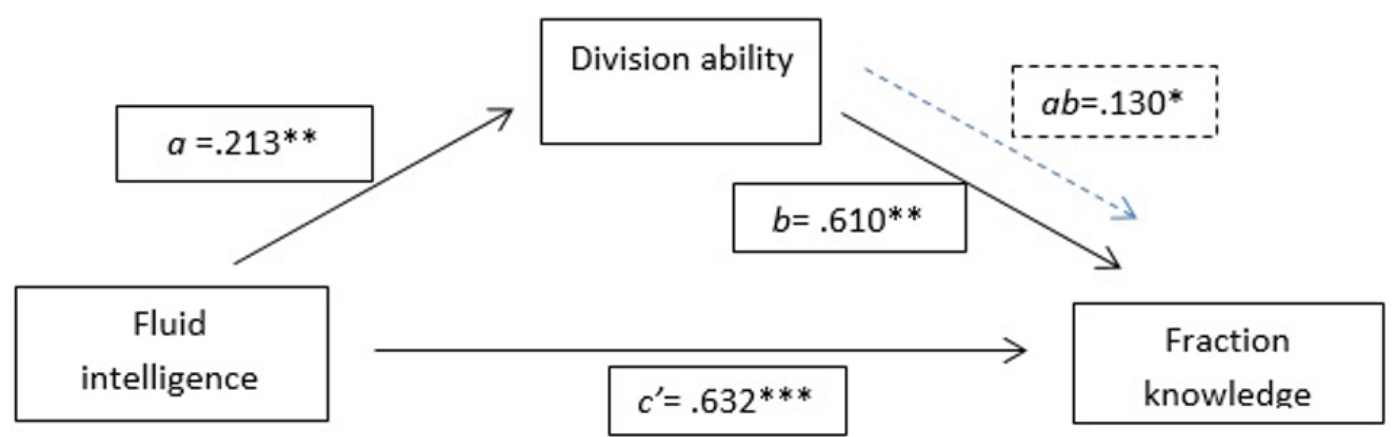

Figure 3. Model testing the mediating effect of division ability on the relationship between fluid intelligence and fraction knowledge

Notes: solid line: direct relation; dashed line: mediated relation

$* * * \mathrm{p}<.001 ; * * \mathrm{p}<.01 ; * \mathrm{p}<.05$

Table 2. Mediation model with dependent variables regressed on mediator and independent variables

\begin{tabular}{cccccccccccc}
\hline & \multicolumn{1}{c}{ Direct effect } & \multicolumn{4}{c}{ Indirect effect } \\
\hline IV & MV & DV & Coef. & SE & Z & Sig. & Sobel coef. & SE & Z & Sig. & \%M \\
\hline SA & DA & FN & 3.5 & 1.04 & 3.37 & .00 & .74 & .50 & 1.47 & .14 & - \\
\hline WM & DA & FN & 1.7 & .74 & 2.3 & .02 & .82 & .36 & 2.28 & .02 & .32 \\
\hline Fl & DA & FN & .63 & .16 & 3.88 & .00 & .13 & .07 & 1.92 & .05 & .17 \\
\hline
\end{tabular}

Notes: IV: independent variable; MV: mediator variable; DV: dependent variable; \%M: proportion of total effect that is mediated; SA: selective attention; DA: division ability; FN: fraction knowledge; WM: working memory; FI: fluid intelligence.

As depicted in Table 2 division ability explained the $32 \%$ of the effect of working memory and the $17 \%$ of the effect of fluid intelligence on fraction knowledge.

\section{Discussion}

The purpose of this study was to evaluate the relationship of selective attention, working memory and fluid intelligence with fraction knowledge in fifth grade, and to analyze if this relation is mediated by division ability. Fraction teaching is mainly developed through problem solving that requires: (a) to select the relevant information inhibiting the interference of irrelevant information, (b) to mentally represent and process number and/or spatial relationships among elements, (c) to produce inferences, (d) to abstract relationships, and (e) to contrast hypothesis. Therefore, we anticipated that selective attention, working memory and fluid intelligence would directly contribute to explain fraction knowledge. Besides, learning mathematics shows a hierarchical structure, in which the construction of simpler concepts enables to develop more complex concepts (Geary et al., 2008). Considering the evidence indicating a relationship of selective attention, working memory and fluid intelligence with the knowledge of division (Hansen et al., 2015; Stelzer et al., 2018; Siegler \& Pyke, 2013; van der Ven et al., 2013), as well as the contribution of this knowledge to learning fractions (Hansen et 
al., 2015), we anticipated that selective attention, working memory and fluid intelligence would be also indirectly related to fraction knowledge, through its effect on division ability.

In general, the results obtained provide evidence in favor of these hypotheses. As regards working memory and fluid intelligence, it was found that both are directly and indirectly related to fraction knowledge. In the case of the indirect relation, division ability mediated the $32 \%$ of the effect of working memory and the $17 \%$ of the effect of fluid intelligence on fraction knowledge. Fractions a/b format express a division. Moreover, the use of fractions as quotient implies representing them as the result of an equal distribution operation (e.g., "If 3 pieces of chocolate were divided in equal parts among 4 friends, each friend will receive $3 / 4$ "). Besides, division constitutes one of the most complex arithmetic operations, whose estimation usually requires to identify and to abstract number relationships (e.g., if $12 / 4=3$, then $1200 / 400=3$ ), as well as to perform a sequential order of steps and to integrate other operations (1200/400=1200-400-400-400). The first case would mainly involve fluid intelligence capacity, while the second would involve working memory. This pattern of relationships would explain why division ability in fourth grade constitutes one of the mechanism by which working memory and fluid intelligence are related to fraction knowledge in fifth grade.

It is important to highlight that in the case of fluid intelligence, the mediator effect of division ability is relatively low (17\%), suggesting that this capacity affects the knowledge of fractions mostly directly. The concept of a fraction includes different meanings referred to a part-whole relationship (a relation between a whole and the amount of parts in which it is being partitioned), a ratio (a relational index between two quantities), a magnitude (a value that can be located on a number line), a quotient (the result of a division), and an operator (an operation applied to another magnitude) (Charalambous \& Pitta-Pantazi, 2007; Kieren, 1976). The curricular design of the Argentine Educative System proposes to reinforce teaching the part-whole meaning, quotient, magnitude and ratio during fifth year, through the inclusion of problems implying number relationships of greater complexity and uncommon fractions. Additionally, in this grade the concept of fractions is introduced as an operator (Ministry of Education, 2011). Fluid intelligence participates in solving new problematic situations and constructing concepts, thus probably explaining the direct contribution of this capacity to the development and integration of the different meanings of fractions.

Furthermore, regarding selective attention, the results indicated that its effect on the fraction knowledge is totally direct, i.e., it is not mediated by division ability. Several studies highlighted that attentional behavior in the classroom constitutes one of the cognitive factors that could better explain fraction understanding (Hansen et al., 2015; Jordan et al., 2013; Resnick et al., 2016). Learning fractions presents a challenge for students, as they should include a series of concepts in conflict with their previous knowledge of natural numbers. The ability of selecting relevant information and preventing the attentional capture produced by distractors might produce a better learning as it enables students to get more benefitted from the teacher's instructions and to solve more easily the problems with fractions.

\section{Theoretical implications}

The results of this study provide empirical evidence in favor of those theoretical models indicating that general cognitive factors and prior math knowledge play a central role in learning more advance math concepts (Geary et al., 2008; LeFevre et al., 2010). Recent updates of such models indicated that the effect of some general cognitive abilities on mathematics learning varies according to the domain of mathematics and to the level of learning (ongoing versus consolidated) (LeFevre et al., 2013; Sowinski, LeFevre, Skwarchuk, Kamawar, Bisanz, \& Smith-Chant, 2015) and it would be mediated in some cases by a simpler math knowledge (Fuchs, Hornburg, \& McNeil, 2016; Geary, Nicholas, Li, \& Sun, 2017; Ye et al., 2016). As regards fraction conceptual knowledge, Vukovic et al. (2014) suggested that attentional capacity in first grade was only related indirectly to fraction conceptual knowledge in fourth grade, through its effect on whole number calculation ability in second grade. Additionally, Ye et al. (2016) reported that the effect of attention, working memory and fluid intelligence during third grade on fraction conceptual knowledge in sixth grade was totally mediated by magnitude reasoning ability in fifth grade. Our study contributes to the literature as it shows that in fifth grade, which represents an intermediate stage in the process of fraction learning in Argentina, fraction knowledge could be explained by the capacities of selective attention, working memory and fluid intelligence, and part of the effect of working memory and fluid intelligence is mediated by division ability. In summary, our results agree with the suggestions of Geary et al.' (2004; 2006) and LeFevre et al.' (2010) models, and provide evidence regarding the specific pattern of relationships between cognitive factors and prior math knowledge on fraction knowledge in fifth grade. 


\section{Practical implications, limitations and future approaches}

The pattern of relationships observed enables to recognize at the beginning of fraction teaching to students with potential difficulties to learn them. It is important to mention that, although the results of this work enable to establish direct and indirect relationships between cognitive abilities and fraction knowledge, the correlational design used does not allow to infer causal relationships among these variables. As far as we know there are no studies contrasting on an experimental basis the effect of variations in the processes of selective attention, working memory and fluid intelligence on the performance in division and, consequently, fraction understanding. Future studies could consider this objective to further understand the effect of cognitive factors on fraction learning.

In spite of the limitations described before, we expect the results of our work to make further progress in understanding the role of cognitive factors and previous concepts in the knowledge of fractions, and, in this way, to contribute to improve teaching practices related to this content.

\section{Disclosure statement}

No potential conflict of interest was reported by the authors.

\section{Notes on contributors}

Florencia Stelzer - Institute of Basic, Applied Psychology and Technology (IPSIBAT), National University of Mar del Plata, National Scientific and Technical Research Council (CONICET), Mar del Plata, Argentina.

María Laura Andrés - Institute of Basic, Applied Psychology and Technology (IPSIBAT), National University of Mar del Plata, National Scientific and Technical Research Council (CONICET), Mar del Plata, Argentina.

Lorena Canet-Juric - Institute of Basic, Applied Psychology and Technology (IPSIBAT), National University of Mar del Plata, National Scientific and Technical Research Council (CONICET), Mar del Plata, Argentina.

Sebastián Urquijo - Institute of Basic, Applied Psychology and Technology (IPSIBAT), National University of Mar del Plata, National Scientific and Technical Research Council (CONICET), Mar del Plata, Argentina.

María Marta Richards - Institute of Basic, Applied Psychology and Technology (IPSIBAT), National University of Mar del Plata, National Scientific and Technical Research Council (CONICET), Mar del Plata, Argentina.

\section{REFERENCES}

Baddeley, A. D., \& Hitch, G.J. (1974). Working memory. In G.A. Bower (Ed.), The Psychology of Learning and Motivation: Advances in Research and Theory (pp. 47-89). New York: Academic.

Baddeley, A. (2012). Working Memory: Theories, Models, and Controversies. Annual Review of Psychology, 63, 1-29. https://doi.org/10.1146/annurev-psych-120710-100422

Bailey, D. H., Siegler, R. S., \& Geary, D. C. (2014). Early predictors of middle school fraction knowledge. Developmental Science, 17(5), 775-785. https://doi.org/10.1111/desc.12155

Baron, R. M., \& Kenny, D. A. (1986). The mediator-moderator distinction in social psychology: Conceptual, strategic, and statistical considerations. Journal of Personality and Social Psychology, 51, 1173-1182.

Booth, J. L., \& Newton, K. J. (2012). Fractions: Could they really be the gatekeeper's doorman?. Contemporary Educational Psychology, 37(4), 247-253. https://doi.org/10.1016/j.cedpsych.2012.07.001

Booth, J. L., Newton, K. J., \& Twiss-Garrity, L. K. (2014). The impact of fraction magnitude knowledge on algebra performance and learning. Journal of Experimental Child Psychology, 118, 110-118. https://doi.org/10.1016/j.jecp.2013.09.001

Brown, B. W. (1991). How gender and socioeconomic status affect reading and mathematics achievement. Economics of Education Review, 10(4), 343-357. 
Canet Juric, L., Introzzi, I., \& Burin, D. (2015). Desarrollo de la Capacidad de Memoria de Trabajo Efectos de Interferencia Inter e Intra Dominio en Niños de Edad Escolar. Revista Argentina de Ciencias del Comportamiento, 7(1), 26-37.

Carpenter, T., Corbitt, M., Kepner, H., Lindquist, M., \& Reys, R. (1980). Results of the second NAEP mathematics assessment: Secondary school. Mathematics Teacher, 73, 329-338.

Cattell, R. B. (1971). Abilities: their structure, growth, and action. Boston: Houghton.

Cayssials, A. (1993). Test de Matrices Progresivas. Manual adaptación Argentina. Buenos Aires: Paidós.

Chan, W.-H., Leu, Y.-C., \& Chen, C.-M. (2007). Exploring group-wise conceptual deficiencies of fractions for fifth and sixth graders in Taiwan. Journal of Experimental Education, 76, 26-57. https://doi.org/10.3200/JEXE.76.1.26-58

Charalambous, C. Y., \& Pitta-Pantazi, D. (2007). Drawing on a theoretical model to study students' understandings of fractions. Educational Studies in Mathematics, 64 (3), 293-316. https://doi.org/10.1007/s10649-006-9036-2

DeWolf, M., Bassok, M., \& Holyoak, K. J. (2015). From rational numbers to algebra: Separable contributions of decimal magnitude and relational understanding of fractions. Journal of Experimental Child Psychology, 13, 72-84. https://doi.org/10.1016/j.jecp.2015.01.013

DeWolf, M., Bassok, M., \& Holyoak, K. J. (2016). A set for relational reasoning: Facilitation of algebraic modeling by a fraction task. Journal of Experimental Child Psychology, 152, 351-366. https://doi.org/10.1016/j.jecp.2016.06.016

Diamond, A. (2013). Executive functions. Annual Review of Psychology, 64, 135-168. https://doi.org/10.1146/annurev-psych-113011-143750

Dirección General de Cultura y Educación (2018). Diseño curricular para la educación primaria: primer ciclo y segundo ciclo (1a ed). La Plata: Dirección General de Cultura y Educación de la Provincia de Buenos Aires. [General Management of Culture and Education (2018). Curricular design for primary education: first cycle and second cycle (1st ed). La Plata: General Management of Culture and Education of Buenos Aires Province.] Retrieved from http://servicios.abc.gov.ar/lainstitucion/organismos/consejogeneral/ disenioscurriculares/primaria/2018/dis-curricular-PBA-completo.pdf

Engle, R. W. (2002). Working memory capacity as executive attention. Current Directions in Psychological Science, 11, 19-23. https://doi.org/10.1111/1467-8721.00160

Engle, R. W., \& Kane, M. J. (2004). Executive attention, working memory capacity, and a two-factor theory of cognitive control. In B. Ross (Ed.), The psychology of learning and motivation (pp. 145-199). New York, NY: Academic Press.

Flotts, M. P., Manzi, J., Jiménez, D., Abarzúa, A., Cayuman, C., \& García, M. J. (2016). Informe de resultados TERCE. Retrieved from http://unesdoc.unesco.org/images/0024/002435/243532S.pdf

Fuchs, M. W., Hornburg, C. B., \& McNeil, N. M. (2016). Specific early number skills mediate the association between executive functioning skills and mathematics achievement. Developmental Psychology, 52(8), 1217-1235. https://doi.org/10.1037/dev0000145

Gabriel, F. C., Coché, F., Szucs, D., Carette, V., Rey, B., \& Content, A. (2013). A componential view of children's difficulties in learning fractions. Frontiers in Psychology, 4, 715. https://doi.org/10.3389/fpsyg.2013.00715

Geary, D. C. (2004). Mathematics and learning disabilities. Journal of Learning Disabilities, 37, 4-15. https://doi.org/10.1177/00222194040370010201

Geary, D. C. (2006). Development of mathematical understanding. In D. Kuhl, R. S. Siegler (Vol. Eds.), Cognition, perception, and language. W. Damon (Gen Ed.), Handbook of child psychology (6th ed., pp. 777-810). New York: John Wiley \& Sons. https://doi.org/10.1002/9780470147658.chpsy0218

Geary, D. C., Boykin, A. W., Embretson, S., Reyna, V., Siegler, R., Berch, D. B., \& Graban, J. (2008). Report of the task group on learning processes. National mathematics advisory panel, reports of the task groups and subcommittees, 4-1. Retrieved from https://www2.ed.gov/about/bdscomm/list/mathpanel/report/ final-report.pdf

Geary, D., Nicholas, A., Li, Y., \& Sun, J. (2017). Developmental Change in the Influence of Domain-General Abilities and Domain-Specific Knowledge on Mathematics Achievement: An Eight-Year Longitudinal Study. Journal of Educational Psychology, 109(5), 680-693. https://doi.org/10.1037/edu0000159 
Hansen, N., Jordan, N. C., Fernandez, E., Siegler, R. S., Fuchs, L., Gersten, R., \& Micklos, D. (2015). General and math-specific predictors of sixth-graders' knowledge of fractions. Cognitive Development, 35, 34-49. https://doi.org/10.1016/j.cogdev.2015.02.001

Hasher, L., Lustig, C., \& Zacks, R. T. (2007). Inhibitory mechanisms and the control of attention. In A. Conway, C. Jarrold, M. Kane, A. Miyake, A., \& J. Towse (Eds.), Variation in working memory (pp. 227-249). New York: Oxford University Press.

Hecht, S. A., \& Vagi, K. J. (2010). Sources of group and individual differences in emerging fraction skills. Journal of Educational Psychology, 102(4), 843-59. https://doi.org/10.1037/a0019824

Hollingshead, A. B. (2011). Four Factor Index of Social Status. Yale Journal of Sociology, 8, 21-52.

Introzzi, I., \& Canet Juric, L. (2013).Tareas de Autorregulación Cognitiva. En Introzzi, I., Canet Juric, L., Comesaña, A., Andres, M. L. \& Richards, M. (2013). Evaluación de la Autorregulación cognitiva y emocional. Presentación de un Programa. Revista Argentina de Ciencias del Comportamiento (suplemento), 1-11.

Jordan, N., Hansen, N., Fuchs, L., Siegler, R., Gersten, R., \& Micklos, D. (2013). Developmental predictors of fraction concepts and procedures. Journal of Experimental Child Psychology, 116(1), 45-58. https://doi.org/10.1016/j.jecp.2013.02.001

Kane, M. J., \& Gray, J. R. (2005). Fluid intelligence. In N. J. Salkind (Ed.), Encyclopedia of Human Development (pp. 528-529). California: Sage Publications.

Kieren, T. E. (1976). On the mathematical, cognitive, and instructional foundations of rational numbers. In R. Lesh (Ed.), Number and Measurement: Papers from a Research Workshop ERIC/SMEAC (pp. 101144). Columbus: OH.

LeFevre, J. A., Berrigan, L., Vendetti, C., Kamawar, D., Bisanz, J., Skwarchuk, S. L., \& Smith-Chant, B. L. (2013). The role of executive attention in the acquisition of mathematical skills for children in Grades 2 through 4. Journal of Experimental Child Psychology, 114(2), 243-261. https://doi.org/10.1016/j.jecp.2012.10.005

LeFevre, J. A., Fast, L., Skwarchuk, S. L., Smith-Chant, B. L., Bisanz, J., Kamawar, D., \& Penner-Wilger, M. (2010). Pathways to mathematics: Longitudinal predictors of performance. Child Development, 81(6), 1753-1767. https://doi.org/10.1111/j.1467-8624.2010.01508.x

Locuniak, M. N., \& Jordan, N. C. (2008). Using kindergarten number sense to predict calculation fluency in second grade. Journal of Learning Disabilities, 41, 451-459. https://oi.org/10.1177/0022219408321126

McGrew, K. S. (2009). CHC theory and the human cognitive abilities project: Standing on the shoulders of the giants of psychometric intelligence research. Intelligence, 37(490), 1-10. https://doi.org/10.1016/j.intell.2008.08.004

McGrew, K. S., \& Evans, J. J. (2004). Internal and external factorial extensions to the Cattell-Horn-Carroll (CHC) theory of cognitive abilities: A review of factor analytic research since Carroll's Seminal 1993 Treatises. St. Cloud, MN: Institute for Applied Psychometrics.

Ministerio de Educación (2011). Núcleos de Aprendizajes Prioritarios Segundo Ciclo EGB / Nivel Primario. Retrieved from http://www.me.gov.ar/curriform/publica/nap/nap_egb2.pdf

Miyake, A., \& Shah, P. (1999). Toward unified theories of working memory: Emerging general consensus, unresolved theoretical issues and future directions. In Miyake, A. \& Shah (Eds.), Models of working memory: Mechanisms of active maintenance and executive control (pp.442-481). Cambridge: Cambridge University Press.

Namkung, J. M., \& Fuchs, L. S. (2015). Cognitive predictors of calculations and number line estimation with whole numbers and fractions among at-risk students. Journal of Educational Psychology, 108(2), 214228. https://doi.org/10.1037/edu0000055

Pascual, L., Galperín, C. Z., \& Bornstein, M. H. (1993). La medición del nivel socioeconómico y la psicología evolutiva: El caso Argentino. Revista Interamericana de Psicologia/Interamerican Journal of Psychology, 27, 59-74.

Peng, P., Namkung, J., Barnes, M., \& Sun, C. (2016). A meta-analysis of mathematics and working memory: Moderating effects of working memory domain, type of mathematics skill, and sample characteristics. Journal of Educational Psychology, 108(4), 455. https://doi.org/10.1037/edu0000079 
Raven, J. C. (1989). Test de Matrices Progresivas para la medida de la capacidad intelectual, de sujetos de 4 a 11 años. Buenos Aires: Paidós. [Raven Progessive Matrices test for IQ assessment in 4 to 11 years old subjets]

Resnick, I., Jordan, N. C., Hansen, N., Rajan, V., Rodrigues, J., Siegler, R. S., \& Fuchs, L. S. (2016). Developmental growth trajectories in understanding of fraction magnitude from fourth through sixth grade. Developmental Psychology, 52(5), 746. https://doi.org/0.1037/dev0000102

Richards, M., Introzzi, I., Zamora, E., \& Vernucci, S. (2017). Analysis of Internal and External Validity Criteria for a Computerized Visual Search Task. A pilot study. Applied Neuropsychology: Child, 110-119. https://doi.org/10.1080/21622965.2015.1083433

Richards, M. M., Vernucci, S., Zamora, E., Canet Juric, L., Introzzi, I., \& Guardia, J. (2017). Contribuciones empíricas para la validez de grupos contrastados de la Batería de Tareas de Autorregulación Cognitiva (TAC). Interdisciplinaria, 34(1), 173-192.

Sautú, R. (1989). Teoría y técnica en la medición del status ocupacional: Escalas objetivas de Prestigio (Documento de Trabajo). Buenos Aires, Argentina: UBA Instituto de Ciencias Sociales.

Siegler, R. S., Duncan, G. J., Davis-Kean, P. E., Duckworth, K., Claessens, A., Engel, M., et al. (2012). Early predictors of high school mathematics achievement. Psychological Science, 23, 691-697. https://doi.org/10.1177/0956797612440101

Siegler, R. S., \& Lortie-Forgues, H. (2017). Hard lessons: Why rational number arithmetic is so difficult for so many people. Current Directions in Psychological Science, 26(4), 346-351. https://doi.org/10.1177/0963721417700129

Siegler, R. S., \& Pyke, A. A. (2013). Developmental and individual differences in understanding of fractions. Developmental Psychology, 94(10), 1994-2004. https://doi.org/10.1037/a0031200

Siegler, R. S., Thompson, C. A., \& Schneider, M. (2011). An integrated theory of whole number and fractions development. Cognitive Psychology, 62, 273-296. https://doi.org/10.1016/j.cogpsych.2011.03.001

Sowinski, C., LeFevre, J.A., Skwarchuk, S. L., Kamawar, D., Bisanz, J., \& Smith-Chant, B. (2015). Refining the quantitative pathway of the Pathways to Mathematics model. Journal of Experimental Child Psychology, 131, 73-93. https://doi.org/10.1016/j.jecp.2014.11.004

Stelzer, F., Introzzi, I., Andres, M. L., Richard's, M., \& Urquijo (2018). Factores cognitivos relacionados con la capacidad de cálculo de división en estudiantes de $4^{\circ}$ año [Cognitive factors related to division ability in fourth grade students]. Actualidades investigativas en educación, 18(1), 1-26.

Thurstone, L. L., \& Yela, M. (2012). CARAS-R. Test de percepción de diferencias-Revisado. Buenos Aires: TEA Ediciones.

Torbeyns, J., Schneider, M., Xin, Z., \& Siegler, R. S. (2014). Bridging the gap: Fraction understanding is central to mathematics achievement in students from three different continents. Learning and Instruction, https://doi.org/10.1016/j.learninstruc.2014.03.002

van der Ven, S.H. G., van der Maas, H. L.J., Straatemeier, M., \& Jansen, B. R. J. (2013). Visuospatial working memory and mathematical ability at different ages throughout primary school. Learning and Individual Differences, 27, 182-192. https://doi.org/10.1016/j.lindif.2013.09.003

Vukovic, R. K., Fuchs, L. S., Geary, D. C., Jordan, N. C., Gersten, R., \& Siegler, R. S. (2014). Sources of individual differences in children's understanding of fractions. Child Development, 85(4), 1461-1476. https://doi.org/10.1111/cdev.12218

Ye, A., Resnick, I., Hansen, N., Rodrigues, J., Rinne, L., \& Jordan, N. C. (2016). Pathways to fraction learning: Numerical abilities mediate the relation between early cognitive competencies and later fraction knowledge. Journal of Experimental Child Psychology, 152, 242-263. https://doi.org/10.1016/j.jecp.2016.08.001 